\title{
IMMERSIONS OF THE CIRCLE AND EXTENSIONS TO ORIENTATION-PRESERVING MAPPINGS ${ }^{1}$
}

\author{
BY ANTONIO O. FARIAS
}

Communicated by Shlomo Sternberg, April 10, 1973

The author extends the results of [1] to the study of immersions of $S^{\mathbf{1}}$ into a 2 -dimensional, oriented manifold $M$, and gives a characterization of those immersions which can be extended to a mapping of the closed disk with nonnegative Jacobian, in terms of geometric operations of growth. These are the analogue of the $T$-operators of [1]; they were introduced and studied by Titus in [2], and consist of displacement along the integral curves of a vector field toward the outside of the curve.

The author wishes to thank Professors Charles Titus and John Little for many helpful and inspiring discussions. In particular, the use of universal coverings was suggested by Professor Little.

1. Preliminaries. The reference for notation and terminology is the author's paper [1]. The following additions and modifications will be used.

The phrases positive extension and positively extendable substitute proper extension and properly extendable, respectively. The fixed orientation of $M$ is the 2 -form $\omega$. If $\Lambda$ is a vector field on $M$, then $\Psi\{\Lambda, z, t\}$ denotes the integral curve of $\Lambda$ through $z$ at time $t=0$. All vector fields considered are divergence-free and have the property that $\Psi\{\Lambda, z, t\}$ is defined for every $(z, t) \in M \times R^{1}$. by

Let $\alpha: S^{1} \rightarrow R^{1}$ be nonnegative; define $T=(\alpha, \Lambda)$ on the set $C^{\infty}\left(S^{1}, M\right)$

$$
(T f)(\theta)=\Psi\left\{\Lambda, f(\theta), \alpha(\theta) \omega_{f(\theta)}\left[\Lambda_{f(\theta)}, f^{\prime}(\theta)\right]\right\} .
$$

Denote by $\mathscr{S}$ the semigroup generated by all such functions under composition; an element of $\mathscr{S}$ will be called a $T$-operator.

AMS (MOS) subject classifications (1970). Primary 57D40, 57D35, 54C20; Secondary 57A05, 55A10, 54C10, 54C05, 57D 12.

Key words and phrases. Normal immersions, extendable mappings, holomorphic mappings, topology of immersions.

1 Part of this research was done while the author was a Visiting Professor at Universidade Federal de Pernambuco (Brazil). 
The effect of a $T$-operator on a curve $f: S^{1} \rightarrow M$ is that of a finite number of growth operations, growth understood in the sense of motion along the integral curves of a vector field toward the outside of the oriented curve $f$.

A mapping $f: S^{1} \rightarrow M$ is degenerate if it can be written as $f=g \circ h$, where $g: R^{1} \rightarrow M$ is a diffeomorphism onto a submanifold of $M$. A $T$ mapping is one which can be written as $T f_{0}$ with $T$ in $\mathscr{S}$ and $f_{0}$ degenerate.

\section{Results.}

THEOREM 1. A normal mapping is extendable if and only if it is a T-mapping.

The if part was essentially proved by Titus [2, Theorem 1]. The only if part follows from Theorem 2 below.

THEOREM 2. Every positively extendable mapping is a T-mapping.

In order to prove Theorem 2 we need to extend the results of [1] to immersions of $S^{1}$ into $M$. This is accomplished by first proving them for the case $M=S^{2}$ and then using universal covering arguments in the general case. In particular, the following results are proved:

THEOREM 3. Every normal mapping has a neighborhood $\mathscr{U}$ in the $C^{1}$ topology of $C^{\infty}\left(S^{1}, M\right)$ with the following properties:

(a) If $g, h \in \mathscr{U}$ there are orientation-preserving diffeomorphisms $\varphi$ of $S^{1}$ and $\psi$ of $M$ such that $g \circ \varphi=\psi \circ h$.

(b) If there is a $g$ in $\mathscr{U}$ which is extendable, then every $h$ in $\mathscr{U}$ is also extendable.

Proposition 1. (1) Every normal, extendable mapping is positively extendable.

(2) The set of positively extendable mappings is open in the $C^{1}$ topology of $C^{\infty}\left(S^{1}, M\right)$.

(3) Let $F$ be a positive extension off. There is a Riemann surface structure on $M$, a properly holomorphic $W: D^{-} \rightarrow M$, an orientation-preserving homeomorphism $H: D^{-} \rightarrow D^{-}$and an open set $U$ containing $S^{1}$ such that $W \circ H=F$ on $U$ and the restriction of $H$ to $U$ is a diffeomorphism into $R^{2}$.

In the proof of part (3) use is made of the following result in general topology.

Proposition 2. Let $X$ be a Hausdorff space and $A$ a closed subspace with empty interior. Let $F: X \rightarrow Y$ be a continuous mapping such that the restriction of $F$ to $X-A$ is a homeomorphism into $Y$. Then, $F(A) \cap$ $F(X-A)=\varnothing . I f$, further, $X$ is locally compact, $Y$ is Hausdorff and $F$ is a 
local homeomorphism from $A$ to $F(A)$, then $F$ is a homeomorphism of $X$ into $Y$.

Details and proofs will appear elsewhere.

\section{REFERENCES}

1. Antonio O. Farias, Orientation-preserving mappings, a semigroup of geometric transformations and a class of integral operators, Trans. Amer. Math. Soc. 167 (1972), 279-289. MR 45 \#4440.

2. Charles J. Titus, Transformation semigroups and extensions to sense-preserving mappings, 1970/71 Preprint Series no. 35, Matematisk Institut, Aarhus Universitet, Denmark.

Department of Mathematics, University of São Paulo, São Paulo, Brazil 